\title{
Three-Dimensional Cell Culture at the Frontiers of in Vitro Cancer Research: Present Perspectives
}

\author{
Soo $\mathrm{LL}^{1 *}$, Zainal AA ${ }^{1}$, Hidetsugu $\mathrm{T}^{2}$, Takabatake $\mathrm{K}^{3}$, Nakano $\mathrm{K}^{3}$, Chai $\mathrm{WL}^{4}$, Nagatsuka $\mathrm{H}^{3}$, Siar \\ $\mathrm{CH}^{1}$
}

${ }^{1}$ Department of Oral and Maxillofacial Clinical Sciences, Faculty of Dentistry, University of Malaya, 50603 Kuala Lumpur, Malaysia

${ }^{2}$ Laboratory of Histopathology, Department of Life Science, Faculty of Science, Okayama University of Science, 1-1 Ridai-cho, Kita-ku, Okayama 700-0005, Japan

${ }^{3}$ Department of Oral Pathology and Medicine, Graduate School of Medicine, Dentistry and Pharmaceutical Sciences, Okayama University, 2-5-1 Shikata-cho, Okayama 700-8525, Japan

${ }^{4}$ Department of Restorative Dentistry, Faculty of Dentistry, University of Malaya, 50603 Kuala Lumpur, Malaysia

\begin{abstract}
In recent years, three-dimensional (3D) in vitro cell culture models have earned great attention, especially in the field of human cancer disease modelling research as they provide a promising alternative towards the conventional two-dimensional (2D) monolayer culture of cells with improved tissue organization. In 2D cell culture systems, the complexity of cells on a planar surface does not accurately reflects the in vivo cellular microenvironment. Cells propagated in 3D cell culture model, on the other hand, exhibit physiologically relevant cell-to-cell interactions and cell-to-extracellular matrix (ECM) interactions, important in maintaining a normal homeostasis and specificity of tissues. This review gives an overview on 2D models and their limitations, followed by 3D cell culture models, their advantages, drawbacks and challenges in present perspectives. The review also highlights the dissimilarities of $2 \mathrm{D}$ and $3 \mathrm{D}$ models and the applicability of 3D models in current cancer research.
\end{abstract}

Keywords: Cell culture, cellular microenvironment, cell-to-cell interaction, cellular morphology, cell polarity, extracellular matrix

\section{INTRODUCTION}

Till recently, cell culture systems that have experienced an accelerated growth are one of the most demanding scientific models available. This in vitro culture system is an indispensable tool for a wide spectrum of applications, ranging from research to industrial perspectives due to its adaptability to the experimental variations that are possible in such culture system (1). The cell culture techniques currently available have undergone a long way of revolution since late 1800s when Wilhelm Roux successfully maintains live neural plate cells from chick embryos in saline buffer for several days (2). Starting from early twentieth century, researchers attempt to develop cell lines by utilizing the cell culture techniques available at that time and a vast 
collection of cell lines is finally blossomed in the midtwentieth century since after the establishment of the HeLa, a first mammalian cell line in 1951. In fact, the establishment of cell lines and the advancement of cell culture techniques are interrelated. This is augmented by the fact that the growth of cell culture techniques is accompanied by the increase in the number of cell lines (1). However, the well-known two-dimensional (2D) cell culture system that was developed over a century ago has raises increasing doubts on its efficiency to maintain the structure and functionality of cultured cells, which is analogous to tissues (3). Thus, three-dimensional (3D) cultures were introduced to improve the fidelity and long term maintenance of cells in an in vitro environment.

\section{D Cell Cultures and Their Limitations}

The $2 \mathrm{D}$ cell culture system is an in vitro static dish culture model that grows monolayer of adherent cells on a flat and rigid substrate, such as artificial plastic or glass, where cultured cells only in contact with each other at their periphery (4). This timehonoured $2 \mathrm{D}$ cell culture system is the most common and classically preferred conventional culture model to reconstitute the in vivo cellular microenvironment (5). Despite the fact that 2D culture models proven to play a pivotal role in biological research over many decades, a multitude of inadequacies and limitations associated have been increasingly recognized. Firstly, the ability of such culture system to emulate the in vivo conditions has been called into questions, because cells in the natural cellular environment are surrounded by extracellular matrix (ECM) and other cells in a complex 3D fashion (6). Secondly, the stress and artificial responses of cells experienced in 2D models due to the cell adaptation to the flat and rigid surfaces in order to establish a favourable environment for optimal cell growth, the morphological and/or functional features of cell in vitro, can significantly alter their ECM proteins expression $(7,8)$. Further, the setting of 2D models also promotes the uneven distribution of receptors on the cell surface and clustering which presumably affect the intercellular communication (8). Such adaptations of cells to the planar 2D culture system are inaccurately recapitulate of the in vivo environment as cells require significant morphological changes which will impair the cellular functions and metabolism to enable the survival in $2 \mathrm{D}$ cultures. Thirdly, the conventional 2D cell culture models lack of the metabolic gradients that present in the in vivo environment caused it to be unsuitable to represent the actual microenvironment, because movements of cells in the in vivo state follow a molecular gradient or chemical signal, important for majority of the biological processes, including cell differentiation, cell fate determination and signal transduction (9, 10). Taken together, these findings emphasize on the inadequacies of 2D models to accurately mimicry of the in vivo microenvironment. Consequently, these shortcomings of 2D models can diminish the cellular properties of 2Dly cultured cells such as the viability, proliferation, differentiation, general cell function and morphology, gene and protein expression and response to external stimuli and drug metabolism, allowing it to support only a limited levels of cell differentiation and in vivo-like functionality (11). As a result, it tends to give an unsatisfactory, misleading and sometimes misinterpreted data for in vivo responses and probably some important discoveries may be missed completely.

The poor predictive power of $2 \mathrm{D}$ cultures in preclinical cell-based drug and toxicity screening assays caused approximately $90 \%$ of promising preclinical drugs that passed in vitro preclinical studies fail in the subsequent clinical trials, and the failure rate is even higher in cancer drugs due to the unsatisfactory of clinical efficacy and undesired safety margin (12-16). Undeniable, immortalized tumour cell lines cultured in 2D models have contributed to the general understanding of tumour growth and progression. However, the inadequacy to effectively model tumour biology and with nearly $95 \%$ of drug candidate attrition rate, 2D culture model is regarded as a poor drug discovery model (17). In the most simplistic view, 2D culture systems imposed physiological constraints on cultured cells; whereby nutrients, oxygen or waste gradients are absent in the culture environment (4). Simultaneously, 2Dly cultured cells are forced to arrange into a monolayer morphology in a planar surface lead to the lacking of interactions between cells and their microenvironment that specifically important in nature tumour. Since 2D cell culture models are lack of realistic complexity and limited culturing of only single cell types, the delay in discovery of successful medical interventions is possible with the involvement of 2D models.

\section{D Culture Models and Their Potentials}

Importantly, the 3D architecture of cellular microenvironment in the in vivo state allows cell-tocell and cell-to-ECM interactions through biochemical and mechanical cues to establish a communication network capable to maintain the specificity and homeostasis of tissues. Over the past two decades, numerous $3 \mathrm{D}$ culture models have developed to overcome the shortfalls of $2 \mathrm{D}$ cell culture system. More recently, 3D cells culture models have gained increasing interest because they are capable to enhance the expression of differentiated function 
and improved tissue organization that is not possible in 2D models (18). In comparison, 3D cell culture approach is a superior in vitro model over $2 \mathrm{D}$ cell culture models that takes into account of the spatial organization of cells. And thus, it allows intricate cell-to-cell interactions and cell-to-ECM interactions in all three dimensions with a dynamic transport system for nutrients, oxygen and discharge of waste products. Hence, cells are able to grow continuously in an undisturbed artificial environment, in contrast to $2 \mathrm{D}$ models where regular trypsinization is required even for normal cell growth. Evidently, 3D models have a greater physiological relevance than $2 \mathrm{D}$ models and this advantage bridges the gap between cellular physiology and the in vitro cell culture systems. In addition, the presence of intercellular communication in 3D models allow cultured cells to adopt a comparatively precise depiction of cell polarization. All in all, artificial 3D culture system is a better cell culture model compared to $2 \mathrm{D}$ culture model. The improved cellular interactions as in 3D models are reminiscent of the actual cellular microenvironment, and hence, the cellular responses and the behaviour of cells cultured in such in vitro setting are comparatively more reflective of the in vivo conditions $(13,19-21)$.

3Dly cultured cells possess morphological and/or functional features that are more accurately reflect the in vivo state (22). An emerging evidence demonstrated the cells grown in the 3D culture system mimic its natural shape and possess cellular heterogeneity similar to the in vivo counterparts. Moreover, 3Dly cultured cells display genotypes that significantly more relevant to in vivo state. Not only that, 3D model also allows the co-cultivation of multiple cell types and so it is more precisely imitates the natural in vivo microenvironment. Such 3D-based cell co-culture system is an ideal in vitro model that is useful in the discovery and understanding of the importance of intercellular communication in cell functions, particularly the role of stromal cells in tumour microenvironment (TME), which play a pivotal part in the development and progression of cancers (23). As evidenced by Weaver et al., breast tumour cells upon the inhibition of $\beta 1$-integrin are capable to revert to a normal epithelial phenotype, whether morphologically or functionally (16). Besides, 3D models have great stability and therefore, cell growth in 3D models are far more stable and have a longer lifespan (24). It was evident that cells cultured in 3D models can last at least up to 4 weeks, in turn, cells cultured in 2D models only last for almost a week due to cell confluency (4). Collectively, 3D model is undoubtedly a promising in vitro model, more appropriate to serves as an invaluable tool for the study of the long-term effects of candidate drugs.
In addition, 3D model provides a more accurate representation of cytoarchitecture and till date, 3D models have been successfully allowed the study of more than 380 cell lines and extensively adopted in stem cell culture and differentiation, cancer cell biology, tissue engineering, cell-based analysis and drug discovery (1). The advancement of 3D models has resulted in significant acceleration of translation research in wide range of medical and cellular projects, including cancer biology, regenerative medicine and tissue engineering, and thus ultimately fuelled the development of novel drugs with low rate of attrition.

Strikingly, 3D-based culture of embryonic stem (ES) cells proved to promote the growth of selforganizing organoid of diverse tissues. Eiraku et al., demonstrated the autonomous formation of the optic cup structure by 3D culture of mouse ES cells under serum-free floating culture of embryoid-bodylike aggregates with quick reaggregation (SFEBq) with an addition of basement-membrane matrix component, Matrigel (25). The generated self-form ES-derived optic cup regulated in a spatiotemporally manner, possessed a distinctive apical-basal polarity and invagination pattern reminiscent of their in vivo counterparts. Suga et al., have produced functioning pituitary tissue from mouse ES cells by 3D cell culture system under SFEBq condition (26). Moreover, the co-induction of hypothalamus and oral ectoderm within ES cell aggregate has successfully recapitulate embryonic pituitary development that shown highly layered structures as observed in vivo and the transplantation of the generated pituitary tissues has a notable capacity in restoration of the systemic glucocorticoid level in hypopituitary mice. Further, Sato and Clevers, have established a R-spondin-based 3D model that successfully grown a single intestinal stem cell, Lgr5-crypt base columnar (CBC) from mice into epithelial organoids with retained original identity (27). The in vivogenerated organoids have self-renewing capacity and cell-type composition closely reminiscent the in vivo state. Further, the engraftation of epithelial organoids into the colons of mice leads to the regeneration of epithelial patches that indifferent from the surrounding recipient epithelium. Other than that, human pluripotent stem cell-derived 3D organoid culture system by Lancaster and Knoblich, successfully developed into a cerebral organoid with various discrete but interdependent brain regions, recapitulate features and development of the human cortical development (28). Methods as demonstrated by Takebe et al., for the generation of transplantable organ bud by co-cultivation of human induced pluripotent stem cells (HiPSCs) with endothelial cells and mesenchymal stem cells (MSCs) showed 
a rapid vascularization and self-organization into functional and tissue-specific structure (19). It is a promising approach with therapeutic potency, in which the transplantation of in-vitro-derived 3D pancreatic condensate successfully treated type I fulminant diabetic mice. Overall, 3D culture system not only serves as a promising model for the study of biology and pathology, but it also a suitable system for the recapitulation of embryonic development and disease that is difficult to recapitulate in mice, for example, microcephaly, possible facilitates the realization of generative medicine and/ or regenerative therapies for organ defects.

\section{Comparision between 2D And 3D Cell Culture Models}

2D models generally grow only single type of cells that forced into a monolayer morphology on a flat surface, such as glass or tissue culture polystyrene plastic flasks (Figure 1A). In contrast, 3D models allow the growth of either single type of cells or co-cultures on pre-coated scaffold/matrix (Figure $1 \mathrm{~B}$ ) or as $3 \mathrm{D}$ aggregates or spheroids within a scaffold/matrix (Figure 1C) or suspended in medium containing matrix in a scaffold-free manner (Figure 1D). The attachment and spreading of cells in 2D models are normally occur within minutes due to its restraint-free nature. In turn, proteolytic degradation of physical environment is necessary in $3 \mathrm{D}$ models prior to the attachment and spreading of cells, which can occur in hours or even days (30). In planar 2D models, cell attachment occurs at only one side of the cell, forcing cells to have limited cellular contact and interactions; whereas cell attachment in 3D models occurs around the entire surface of the cells (31). Such setting of $3 \mathrm{D}$ cell culture models allows the existence of cellto-cell interactions and cell-to-ECM interactions and hence enable the crosstalk between cultured cells and their surrounding $3 \mathrm{D}$ environment as in the in vivo conditions. In fact, the interactions that existed in $3 \mathrm{D}$ culture models contributed to the fundamental differences as observed in 2D and 3D models. These interactions are important during in vitro culturing to achieve the in vivo-like structural organization and connectivity, able to limit or even diminish cell morphology and functional features of cell, including cell viability, differentiation, proliferation, gene and protein expression (6).

Regardless of the cell line and matrix dependence, the rate of proliferation of 2Dly and 3 Dly cultured cells are usually differed, mainly due to their dissimilarity in spatial organization. Moreover, a tremendous amounts of cell line cultured in $3 \mathrm{D}$ models exhibit a reduced proliferation rate in comparison to those 2Dly cultured cell lines (3236). Generally, the proliferation rate of 3Dly cultured cells is more accurately mimicry of the rate of growth of cells in the in vivo environment than the 2Dly cultured cells (34). Besides, the dissimilarity in cell morphology is obvious when comparing $2 \mathrm{D}$ and 3D cultures. In fact, appropriate cell polarity is another important physiological attribute conferred by the cellular interaction, including cell-to-cell and cell-to-ECM interactions that possible in 3D models. With the absence of cellular contact in unnatural 2D models, it resulted in the default apical-basal polarity and alteration of cellular morphology that ultimately influence the cellular function (31). The cell morphology such as cytoskeletal organization and cell adhesions of 3D cultures are shown to be much more closely resemble to their in vivo counterparts due to the relatively precise cell polarization, compared to 2D cultures with only partial polarization. In addition, the setting of $2 \mathrm{D}$ models created a non-physiologically uniform environment, where uniformly rich oxygen, nutrients and growth factors are provided to all cells in the culture (37). Unlike conventional 2D monolayer cell culture models, oxygen, nutrients and waste gradients are present in the 3D models. Moreover, the restricted nutrition and oxygenation environment encountered by 3Dly cultured cells are actually emulate the microenvironment as in the in vivo tissues (38). With similar physiological environment of nutrients and gaseous exchange restriction, the cellular heterogeneity present in $3 \mathrm{D}$ models resembles to the in vivo tissues, where both comprises of cells at various stage, including proliferating, apoptotic, necrotic, quiescent and hypoxic cells $(39,40)$. In contrast, cellular heterogeneity is absent in 2D models and this system is generally composed of only flat and stretched cells that exhibit a relative uniform proliferation across the surface due to the consistency of medium exposure (6). These differences strongly suggested that $3 \mathrm{D}$ model is an excellent model system that able to supports varying degrees of cell complexity and functionality as in the natural environment compared to $2 \mathrm{D}$ model. The generation of microenvironment that mimic the physiological conditions enable the cellular response of 3Dly cultured cells to drug treatments to be more reflective of the in vivo conditions (41-43). 

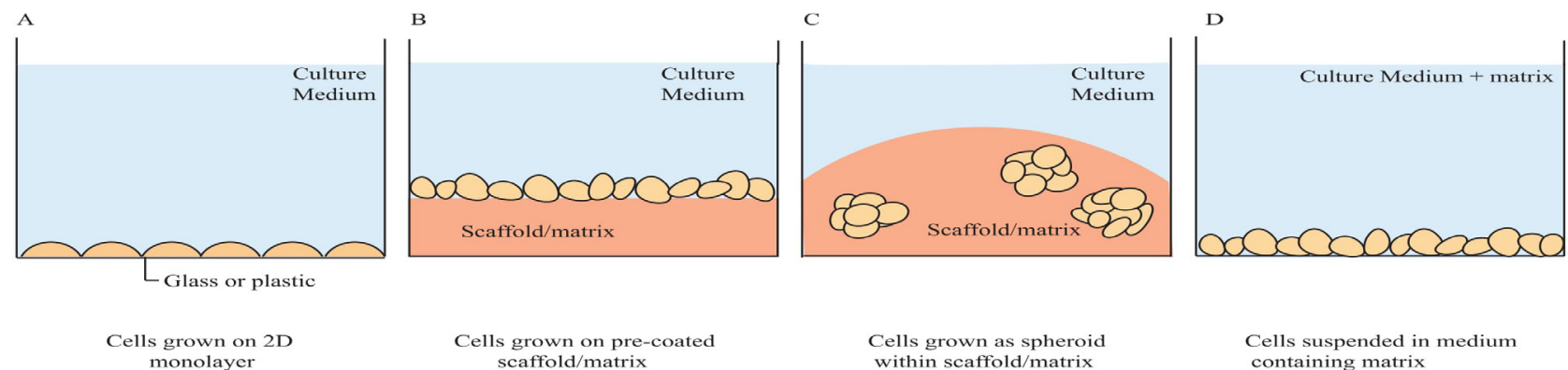

Figure 1: Schematic diagram of two-dimensional (2D) cell culture (A) and three-dimensional (3D) cell culture systems: cells grown on pre-coated scaffold//matrix (B), grown as spheroid within scaffold/matrix (C) and scaffold/matrix-fee manner in medium containing matrix

Table 1: Two-dimensional (2D) versus three-dimensional (3D) cell culture systems

\begin{tabular}{|c|c|c|}
\hline Variables & $2 \mathrm{D}$ & $3 \mathrm{D}$ \\
\hline Morphology & $\begin{array}{l}\text { Flat and stretched cells forced into a } \\
\text { monolayer on a flat surface (6). }\end{array}$ & $\begin{array}{l}\text { Cells maintain its normal shape and structure } \\
\text { into a 3D aggregates or spheroids ( } 7) \text {. }\end{array}$ \\
\hline Cell attachment & $\begin{array}{l}\text { Normally occur within minutes at only one } \\
\text { side of the cell (30). }\end{array}$ & $\begin{array}{l}\text { Can occur in hours or even days around the } \\
\text { entire surface of the cells (30). }\end{array}$ \\
\hline Proliferation rate & $\begin{array}{l}\text { Cells proliferate at a relatively uniform rate } \\
\text { but often at a faster rate than in vivo (6). }\end{array}$ & $\begin{array}{l}\text { Accurate representation of cell growth rate in } \\
\text { vivo (34). }\end{array}$ \\
\hline Cell polarity & $\begin{array}{l}\text { Cells displayed a default apical-basal } \\
\text { polarity with only partial polarization ( } 4 \text {, } \\
\text { 31). }\end{array}$ & Precise depiction of cell polarization (4). \\
\hline $\begin{array}{l}\text { Cellular } \\
\text { heterogeneity }\end{array}$ & Cells generally in the same stage (6). & $\begin{array}{l}\text { Spheroids comprises of cells in various } \\
\text { stage, such as quiescent, proliferating, } \\
\text { apoptotic, necrotic and hypoxic cells }(39,40) \text {. }\end{array}$ \\
\hline $\begin{array}{l}\text { Cell culture } \\
\text { environment }\end{array}$ & $\begin{array}{l}\text { Non-physiologically uniform environment } \\
\text { provides rich oxygen, nutrients and growth } \\
\text { factors to all cells uniformly ( } 37) \text {. }\end{array}$ & $\begin{array}{l}\text { Restricted oxygenation, nutrition environment } \\
\text { and waste gradient are accurately emulate } \\
\text { the natural cellular environment ( } 38) \text {. }\end{array}$ \\
\hline
\end{tabular}

\section{Applications Of 3D Cell Culture Model in Cancer Research}

3D culture models have been a subject of interest since it exhibits a hierarchical structure and cellular heterogeneity, enable a more precise representation of in vivo cell morphology and function. With this advantage, 3D models emerge as a suitable model in revealing of novel and unanticipated visions into tumorigenesis mechanism, significantly accelerate the research in cancer biology. Frequently, 3D culture model is used extensively in studying of the expression of hallmark characteristics of tumour cells, including uncontrolled proliferation, expression of growth signals, transition of cells, anti-apoptosis, invasion, angiogenesis and metastasis (44). 2D cell culture models genuinely contributed to a multitude of knowledge in cancer biology, however, the inability of 2Dly cultured cells to form a multidimensional structure simply do not reflects the in vivo cellular microenvironment. In comparison, 3D cultures of tumour cells with customized microenvironments are a scientifically-rigorous culture system that better mimic the cells growing within the living tumours.

Myungjin Lee et al., cultured 31 epithelial ovarian cancer (EOC) cell lines in 3D by using polyhydroethylamethacrylate-coated tissue culture plastics for 14 days (45). The 3Dly cultured EOC cell lines formed multicellular aggregate structures (MCA) and restored the histological differentiation of the primary tumours that was absent in 2D cultures. Moreover, the molecular changes in 3Dly cultured EOC cell lines much more closely reflect the molecular features of the primary tumours compared to 2Dly cultured cells. Whilst, Jaganathan et al., have developed an in vitro 3D heterogeneous tumour model for breast cancer by utilising magnetic levitation system in order to recapitulate the in vivo breast cancer cell-fibroblast interactions (46). The proposed 3D co-culture model proves to be a useful model in dissection of the influences of tumour microenvironment on tumour cell behaviours. It is said to be an advantageous model due to its capability 
to form a larger sized breast tumour model in short time, manipulation on the tumour compositions and densities, and more accurately resemble the in vivo tumour microenvironment, and thus it allows a better understanding of tumour biology. Besides, the 3D coculture model comprises ameloblastoma-associated fibroblasts (AAFs) and gingival fibroblasts (GFs) with head and neck squamous cell carcinoma cell lines separately developed by Chantravekin and Koontongkaew, provided a valuable tool to reveal the tendency of AAFs in stimulate the proliferation and invasion of tumour cells more than GFs, putatively through TGF- $\beta$ expression (47). And, the 3D laminin rich ECM models by Cichon et al., further embark on the importance of cell-ECM interactions in cellular function (48). 3Dly cultured lung cancer cells displayed an observable development of multicellular structures that reflective of the phenotypic alterations controlling of the cancer cell malignancy.

Furthermore, the physiological relevance of 3D culture model enable it to be a representative of a promising in vitro cell culture system for the study of cancer-relevant patterns of cellular processes. By using 3D culture models, Harma et al., successfully analysed the growth modes, migration and invasion of prostate cancer cells, because the nature of $3 D$ models allowed the monitoring and modulation of invasive processes of tumour cells in organotypic environment (49). In addition, it is evident that 3Dly cultured prostate cancer cells are more relevant to the tumour cell biology than those cultured in 2D model, enable it to make a significant contribution to the finding of polarized epithelial structures which resemble the morphology, biochemistry and invasive processes of tumours as in the in vivo conditions. Bokhari et al., have demonstrated the capability of $3 \mathrm{D}$ models in narrowing the gap between in vitro cell culture systems and the cellular physiology (24). The HepG2 liver cells cultured in 3D model shown to exhibits a greater viability, maintained structural integrity and less susceptible to apoptosis or necrosis even at a high level of cytotoxin compared to their 2D counterparts. Moreover, HepG2 cells grown on $3 \mathrm{D}$ model performed a normal metabolic activity and cultured cells occupied a 3D environment allowed them to interact with adjacent the cells in order to maximise their surface area as in the natural cellular environment. In contrast, HepG2 cells displayed a heterogeneous and disorganised appearance with flat extended structures on 2D substrate, and appeared unhealthy with some of the cells rounding up and some disintegrating starting from day 14 . In essence, these findings embrace the potentials of 3D model to arise as an excellent model system that resemble more closely of the in vivo microenvironment.

\section{Applications in Oral Cancer}

2D models are a valuable tool in oral cancer research that have contributed a tremendous amount of knowledge underlying the mechanism of cancer, however, the physical and biochemical features of the solid tumour mass do not achieve in such monolayer cultures. Owing to the limitations of 2D models, 3D models appear to be a promising alternative that bridge the gap between $2 \mathrm{D}$ and animal studies. At present, the 3D models of normal oral mucosa are well established and the co-culture model developed has been adapted by researchers to recapitulate oral dysplasia or cancer $(50,51)$.

The bidirectional interactions between cells and their microenvironment are important for the normal tissue homeostasis as well as tumour growth (52). In native tumours, the communication between tumour cells and the associated stroma in TME is achieved via cell junctions, receptors, hormones or soluble factors are critical in sustaining the tumour growth, invasion and metastasis. Li et al., have demonstrated the role of cancer-associated fibroblasts (CAFs) in promoting the growth, proliferation, mobility, invasion and epithelial-mesenchymal transition (EMT) of oral tongue squamous cancer cell lines (OTSCC) in a 3D co-culture model (53). The model provided the ability to dissect the intercellular communication between CAFs and OTSCC cell lines in order to determine the factors manipulating the behaviour of OTSCC.

It is well known that the crosstalk between the stromal microenvironment and the tumour cells plays a significant role in activation of the signalling pathways that can promote tumour invasion and progression. In order to examine the feasibility of 3D models in studying oral cancer cell invasion, Duong et al., developed a 3D construct that included an epithelial component of oral squamous carcinoma cells (OSCC) that seeded atop of a layer of connective tissue containing oral mucosa fibroblasts, separated by a reconstituted basement membrane. By utilizing the constructed 3D models, the invasion of the OSCCs into the underlying basement membrane and the connective tissue stroma, as well as their associated mechanism can be observed in varying culture conditions and treatments at different time intervals with ease (54). Whilst, the $3 \mathrm{D}$ human oral squamous cell carcinoma (OSCC3) model using synthetic poly(lactide-co-glycolide) (PLG) scaffolds established by Fischbach et al., (55) able to restored the histological characteristics of primary tumours. Besides, 3D PLG cultured OSCC3 cells transitioned to a fibroblastic morphology, acquired migratory phenotypes and upregulated mesenchymal biomarkers suggests that tumour cells cultured in 3D PLG microenvironment exhibit 
enhanced invasive potential. And, the increased concentration of fibronectin and enhanced human umbilical vein endothelial cell (HUVEC) proliferation in 3D PLG culture of OSCC-3 cells linked to their enhanced angiogenic capacity in relative to their $2 \mathrm{D}$ counterparts. Overall, 3D PLG culture enhanced the invasion capacity and tumour progression of OSCC3 cells.

In the study conducted by Eriksson et al., aimed to examine the effect of co-culture environment on ameloblastoma (AM-1) cells within a collagen construct, the AM-1 construct and the bone-like construct composed of human osteosarcoma (HOS) cells and Bio-Oss granules within a collagen construct was joined together with an acellular support gel (56). The established co-culture model demonstrated the presence of HOS cells in attracting the migration of AM-1 cells through the bone-like part of the construct is then further quantified by Transwell inserts. Further, the presence of HOS cells in the co-culture construct upregulated the RANKL expression of AM-1 cells, but the presence of AM-1 cells downregulated the OPG and NF-KB expression of bone-like construct increases the rate of bone resorption. In order to analyse the role of neutrophils in invasion potential of OSCC, Glogauer et al., established a direct and indirect co-cultures of human peripheral blood neutrophils and UMSCC47 cells (OSCC cell line) in Matrigel-coated Transwell support (57). The established co-cultures model revealed that the presence of neutrophils increases the invasiveness of UMSCC47 cells even without direct contact via the activation of invadopodia formation and matrix degradation to enable the oral cancer cells to invade into the surrounding tissues.

\section{Drawbacks of 3D Model}

On the downside, even the most impressive and advanced $3 \mathrm{D}$ models fail to exactly recapitulate the morphological characteristics including tissue-tissue interfaces, spatiotemporal gradients of oxygen, nutrients and chemicals, and the mechanically active microenvironmental cues of a living organ that are important for their general function (58). Scaffold/ matrix-based $3 \mathrm{D}$ culture systems possess a major technical challenge in terms of the scaffolds/matrix used in the system. Of the various 3D models developed, some uses matrices of animal origin components which may lead to the implementation difficulties in clinical work, whereas some uses matrices made from tissue such as basement membrane extracts and these matrices potentially contain unknown or undesired components, such as growth factors and viruses. Other than that, some matrices that allow for cell attachment in culture system may not be able to effectively remove the cells attached on the matrices and thus it affects the assay development (59). As example, collagen hydrogels and Matrigel have to be handled well to maintain its density and low viscosity respectively in order to allow the manipulation and mixing of cells in 3D culture system. Hence, the choice of scaffolds or matrix, taking into the consideration of porosity, pore distribution and exposed surface area of the scaffolds play a critical role in the distribution and penetration of cells within the scaffold volume, which will significantly affect the architecture of the generated ECM. Whilst, the $3 \mathrm{D}$ cell cultures in gels must be control with caution, because the culture conditions, including the culture $\mathrm{pH}$ and temperature are very important for the functional and effectiveness of a model. These technical difficulties embark on the user-unfriendly nature of the 3D model. Besides, there is a poor level of reproducibility and consistency between sets of experiment and difficulties in cell extraction from the bio-scaffold for analysis due to the increased size and tortuosity of the scaffold. Also, the creation of $3 \mathrm{D}$ cell construct can be very difficult and laborious due to the requisition of many different components. Undoubtedly, 3D model provided a more accurate system for the generation of microenvironment that mimic physiological conditions, however, it has a limited capacity in scaling of cell culture system and also the post-culturing processing of the system. In accordance to the size of the bioscaffold and transparency of material used, the imaging of 3D model may be difficult depends on the microscope depth. It is noted that the frequent use of 3D culture models in different research areas is generally followed by the technical challenges in terms of sensitivity, performance, and compatibility of 3D models with high-throughput screening instruments. However, the assays currently available for the investigation of cellular responses to drug interactions, including cell-to-cell and cell-to-matrix interactions, cell migration and dose dependent cell viability are not optimized for the increasingly sophisticated 3D models. Therefore, optimization is highly required for specifically prepared $3 D$ culture systems to suit for the most of the experimental approaches (4).

\section{Challenges of 3D Model}

Even though 3D models are useful in reconstitute the actual in vivo cellular environment, in which cell-based experiments can be performed very accurately, 3D models have yet to replace the traditional 2D models on a large scale. The limitations of existing 3D culture models in terms of its scalability, sensitivity, reproductivity, and compatibility with the high- 
throughput screening instruments, remained as major challenge for 3D model. Other than that, 3D models also faced challenges on limited data that addressed the mechanism of cell differentiation, drug interaction, and cell signalling. Therefore, the limited knowledge on the functionality of $3 D$ models diminishes the confidence for adoption, although number of publications is increasing rapidly. Further, 3D model also encountered challenges in designing different experimental assays with distinct characteristics for different cell types or nature of applications. Hence, a universal standardized 3D culture system with systematic optimization and characterization is strongly necessary to fully utilized the benefits of 3D culture models in the understanding of the in vivo cellular behaviours which ultimately facilitates the development of various biological research.

\section{Acknowledgements}

This study was jointly supported by the Ministry of Higher Education Malaysia Fundamental Research Grant Scheme (FP032-2015A), University of Malaya Postgraduate Research Grant Scheme PG3362016A, JSPS KAKENHI Grant No. 16K11441, 16K20577 and 26462783.

\section{References}

1. Ravi M, Paramesh V, Kaviya S, Anuradha E, Solomon F. 3D cell culture systems: advantages and applications. J Cell Physiol. 2015; 230(1): 16-26.

2. Rodríguez-Hernández CO, Torres-García SE, Olvera-Sandoval C, Ramírez-Castillo FY, Muro AL, Avelar-Gonzalez FJ, et al. Cell culture: history, development and prospects. Int J Curr Res Aca Rev. 2014; 2(12): 188-200.

3. Shamir ER, Ewald AJ. Three-dimensional organotypic culture: experimental models of mammalian biology and disease. Nat Rev Mol Cell Biol. 2014; 15(10): 647-64.

4. Antoni D, Burckel H, Josset E, Noel G. Threedimensional cell culture: a breakthrough in vivo. Int J Mol Sci. 2015; 16(3): 5517-27.

5. Hess MW, Pfaller K, Ebner HL, Beer B, Hekl D, Seppi T. 3D versus 2D cell culture: implications for electron microscopy. Methods Cell Biol. 2010; 96: 649-70.

6. Edmondson R, Broglie JJ, Adcock AF, Yang L. Three-dimensional cell culture systems and their applications in drug discovery and cellbased biosensors. Assay Drug Dev Technol. 2014; 12(4): 207-18.
7. Knight E, Przyborski S. Method for simple and routine three-dimensional cell culture. Cellular in vitro testing: methods and protocols: Pan Stanford Publishing; 2014: 149-168.

8. Zhang S, Gelain F, Zhao X. Designer selfassembling peptide nanofiber scaffolds for $3 \mathrm{D}$ tissue cell cultures. Semin Cancer Biol. 2005; 15(5): 413-420.

9. Alberts B, Johnson A, Lewis J, Morgan D, Raff M, Roberts K, et al. (2002). Molecular biology of the cell, 6th ed. Garland Science: New York.

10. Lodish H, Berk A, Zipursky SL, Matsudaira P, Baltimore D, and Darnell JE. Molecular Cell Biology, 4th ed. W. H. Freeman, New York. 2000.

11. Sanyal S. Culture and assay systems used for 3D cell culture. Corning. 2014; 9: 1-18.

12. Bhadriraju $\mathrm{K}$, Chen CS. Engineering cellular microenvironments to improve cell-based drug testing. Drug Discov Today. 2002; 7(11): 612620.

13. Birgersdotter A, Sandberg R, Ernberg I. Gene expression perturbation in vitro--a growing case for three-dimensional (3D) culture systems. Semin Cancer Biol. 2005; 15(5): 405-12.

14. Hopkins AL. Network pharmacology: the next paradigm in drug discovery. Nat Chem Biol. 2008; 4(11): 682-90.

15. Kola I. The state of innovation in drug development. Clin Pharmacol Ther. 2008; 83(2): 227-230.

16. Weaver VM, Petersen OW, Wang F, Larabell CA, Briand P, Damsky C, et al. Reversion of the malignant phenotype of human breast cells in three-dimensional culture and in vivo by integrin blocking antibodies. J Cell Biol. 1997; 137(1): 231-245.

17. Hutchinson L, Kirk R. High drug attrition rateswhere are we going wrong?. Nat Rev Clin Oncol. 2011; 8(4): 189-190.

18. Pampaloni F, Reynaud EG, Stelzer EH. The third dimension bridges the gap between cell culture and live tissue. Nat Rev Mol Cell Biol. 2007; 8(10): 839-845.

19. Justice BA, Badr NA, Felder RA. 3D cell culture opens new dimensions in cell-based assays. Drug Discov Today. 2009; 14(1-2): 102-107.

20. Reininger-Mack $A$, Thielecke $H$, Robitzki AA. 3D-biohybrid systems: applications in drug screening. Trends Biotechnol. 2002; 20(2): 5661.

21. Sun T, Jackson S, Haycock JW, MacNeil S. Culture of skin cells in $3 D$ rather than $2 D$ 
improves their ability to survive exposure to cytotoxic agents. J Biotechnol. 2006; 122(3): 372-381.

22. Vinci M, Gowan S, Boxall F, Patterson L, Zimmermann M, Court W, Lomas C, Mendiola M, Hardisson D, Eccles SA. Advances in establishment and analysis of three-dimensional tumor spheroid-based functional assays for target validation and drug evaluation. BMC Biol. 2012; 10: 29.

23. Wang $X$, Sun $L$, Maffini MV, Soto $A$, Sonnenschein C, Kaplan DL. A complex 3D human tissue culture system based on mammary stromal cells and silk scaffolds for modeling breast morphogenesis and function. Biomaterials. 2010; 31(14): 3920-3929.

24. Bokhari M, Carnachan RJ, Cameron NR, Przyborski SA. Culture of HepG2 liver cells on three dimensional polystyrene scaffolds enhances cell structure and function during toxicological challenge. J Anat. 2007; 211(4): 567-576.

25. Eiraku M, Takata N, Ishibashi H, Kawada M, Sakakura E, Okuda S, et al. Self-organizing optic-cup morphogenesis in three-dimensional culture. Nature. 2011; 472(7341): 51-56.

26. Suga $H$, Kadoshima T, Minaguchi M, Ohgushi M, Soen M, Nakano T, et al. Self-formation of functional adenohypophysis in threedimensional culture. Nature. 2011; 480(7375): 57-62.

27. Sato $T$ and Clevers $H$. Growing self-organizing mini-guts from a single intestinal stem cell: mechanism and applications. Science. 2013; 340(6137): 1190-1194.

28. Lancaster MA and Knoblich JA. Organogenesis in a dish: modeling development and disease using organoid technologies. Science. 2014; 345(6194): 1247125 - 1247129.

29. Takebe T, Enomura M, Yoshizawa E, Kimura M, Koike $\mathrm{H}$, Ueno $\mathrm{Y}$, et al. Vascularized and complex organ buds from diverse tissues via mesenchymal cell-driven condensation. Cell Stem Cell. 2015; 16(5): 556-565.

30. Khetan S, Burdick JA. Patterning network structure to spatially control cellular remodeling and stem cell fate within 3-dimensional hydrogels. Biomaterials. 2010; 31(32): 82288234.

31. Baker BM, Chen CS. Deconstructing the third dimension: how 3D culture microenvironments alter cellular cues. J Cell Sci. 2012; 125(13): 3015-3024.
32. Chitcholtan K, Sykes PH, Evans JJ. The resistance of intracellular mediators to doxorubicin and cisplatin are distinct in 3D and 2D endometrial cancer. J Transl Med. 2012; 10(1): 38.

33. Fallica B, Maffei JS, Villa S, Makin G, Zaman M. Alteration of cellular behavior and response to PI3K pathway inhibition by culture in 3D collagen gels. PLoS One. 2012; 7(10): e48024.

34. Gurski LA, Petrelli NJ, Jia X, Farach-Carson MC. 3D matrices for anti-cancer drug testing and development. Oncology. 2010(25): 20-25.

35. Maria OM, Maria O, Liu Y, Komarova SV, Tran SD. Matrigel improves functional properties of human submandibular salivary gland cell line. Int J Biochem Cell Biol. 2011; 43(4): 622-631.

36. Luca AC, Mersch S, Deenen R, Schmidt S, Messner I, Schafer KL, et al. Impact of the 3D microenvironment on phenotype, gene expression, and EGFR inhibition of colorectal cancer cell lines. PLoS One. 2013; 8(3): e59689.

37. Huang H, Ding Y, Sun XS, Nguyen TA. Peptide hydrogelation and cell encapsulation for 3D culture of MCF-7 breast cancer cells. PLoS One. 2013; 8(3): e59482.

38. Lin RZ, Chang HY. Recent advances in threedimensional multicellular spheroid culture for biomedical research. Biotechnol J. 2008; 3(910): 1172-1184.

39. Khaitan D, Chandna S, Arya M, Dwarakanath B. Establishment and characterization of multicellular spheroids from a human glioma cell line; Implications for tumor therapy. J Transl Med. 2006; 4: 12.

40. Kim JB. Three-dimensional tissue culture models in cancer biology. Semin Cancer Biol. 2005; 15(5): 365-377.

41. Lee J, Cuddihy MJ, Kotov NA. Threedimensional cell culture matrices: state of the art. Tissue Eng Part B Rev. 2008; 14(1): 61-86.

42. Shield K, Ackland ML, Ahmed N, Rice GE. Multicellular spheroids in ovarian cancer metastases: Biology and pathology. Gynecol Oncol. 2009; 113(1): 143-148.

43. Zietarska M, Maugard CM, Filali-Mouhim A, Alam-Fahmy M, Tonin PN, Provencher DM, et al. Molecular description of a 3D in vitro model for the study of epithelial ovarian cancer (EOC). Mol Carcinog. 2007; 46(10): 872-885.

44. Koontongkaew S. The tumor microenvironment contribution to development, growth, invasion 
and metastasis of head and neck squamous cell carcinomas. J Cancer. 2013; 4(1): 66-83.

45. Myungjin Lee J, Mhawech-Fauceglia P, Lee N, Cristina Parsanian L, Gail Lin Y, Andrew Gayther $\mathrm{S}$, et al. A three-dimensional microenvironment alters protein expression and chemosensitivity of epithelial ovarian cancer cells in vitro. Lab Invest. 2013; 93(5): 528-542.

46. Jaganathan $H$, Gage J, Leonard F, Srinivasan S, Souza GR, Dave B, et al. Three-dimensional in vitro co-culture model of breast tumor using magnetic levitation. Sci Rep. 2014; 4: 6468.

47. Chantravekin Y, Koontongkaew S. Effects of ameloblastoma-associated fibroblasts on the proliferation and invasion of tumor cells. Journal of Cancer Research and Therapeutics. 2014; 10(4): 1082-1087.

48. Cichon MA, Gainullin VG, Zhang Y, Radisky DC. Growth of lung cancer cells in threedimensional microenvironments reveals key features of tumor malignancy. Integr Biol. 2012; 4(4): 440-448.

49. Harma V, Virtanen J, Makela R, Happonen A, Mpindi JP, Knuuttila M, et al. A comprehensive panel of three-dimensional models for studies of prostate cancer growth, invasion and drug responses. PLoS One. 2010; 5(5): e10431.

50. Marsh D, Suchak K, Moutasim KA, Vallath $S$, Hopper C, Jerjes W, et al. Stromal features are predictive of disease mortality in oral cancer patients. J Pathol. 2011; 223(4): 470-481.

51. Gaballah K, Costea DE, Hills A, Gollin SM, Harrison P, Partridge $M$. Tissue engineering of oral dysplasia. J Pathol. 2008; 215(3): 280-289.

52. Alonso-Nocelo M, Abuin C, Lopez-Lopez R, de la Fuente M. Development and characterization of a three-dimensional co-culture model of tumor T cell infiltration. Biofabrication. 2016; 8(2): 025002.

53. Li H, Zhang J, Chen S-W, Liu L-I, Li L, Gao F, et al. Cancer-associated fibroblasts provide a suitable microenvironment for tumor development and progression in oral tongue squamous cancer. J Transl Med. 2015; 13(1): 198.

54. Duong HS, Le AD, Zhang Q, and Messadi DV. A novel 3-dimensional culture system as an in vitro model for studying oral cancer cell invasion. Int J Exp Pathol. 2005; 86(6): 365-374.

55. Fischbach $\mathrm{C}$, Chen R, Matsumoto T, Schmelzle T, Brugge JS, Polverini PJ, et al. Engineering tumors with 3D scaffolds. Nat Methods. 2007; 4(10): 855-860.

56. Eriksson TM, Day RM, Fedele S, Salih VM. The regulation of bone turnover in ameloblastoma using an organotypic in vitro co-culture model. J Tissue Eng. 2016; 7: 2041731416669629.

57. Glogauer JE, Sun CX, Bradley G, Magalhaes MA. Neutrophils increase oral squamous cell carcinoma invasion through an invadopodiadependent pathway. Cancer Immunol Res. 2015; 3(11): 1218-1226.

58. Huh D, Hamilton GA, Ingber DE. From 3D cell culture to organs-on-chips. Trends Cell Biol. 2011; 21(12): 745-754.

59. Fawcett T. Matrices for Cell Culture - How to develop healthy attachment. What are the differences, advantages/disadvantages to 3-D matrices?. http://cellculturedish.com/askthe-expert/matrices-for-cell-culture-how-todevelop-healthy-attachment/.

\section{Corresponding author:}

\section{Soo Leng Lee}

Department of Oral and Maxillofacial Clinical

Sciences,

Faculty of Dentistry,

University of Malaya,

Kuala Lumpur,

50603 Kuala Lumpur

Malaysia.

Tel: +603-7967 4807

Fax: +603-7967 4534

Email: leng527@siswa.um.edu.my 